\title{
International Comparison of Long-Term Care: the Need for Resident-Level Classification
}

\author{
Brant E. Fries, PhD, ${ }^{*}$ Gunnar Ljunggren, $M D, \dagger$ and Bengt Winblad, $M D \ddagger$
}

Differences between long-term care facilities in Stockholm (1134 residents) and New York (95,000 residents statewide) were examined. The comparison employed a resident classification system, Resource Utilization Groups (RUG-II), which links individuals' characteristics to resource use. Distributions of Activity of Daily Living functionality and RUG-II categories demonstrated signif- icant differences between these two populations, with the Stockholm facilities more akin to the heavier care skilled nursing facilities in New York. These differences may indicate different uses of long-term care beds in the United States and Sweden and demonstrate the need for resident-level classification systems in cross-national studies. J Am Geriatr Soc 39:10-16, 1991



ong-term care (LTC), and principally care of the elderly, is one of the fastest growing segments of health care ${ }^{1,2}$ and health care costs ${ }^{3}$ as we approach the next century. As a result, research has focused both on understanding the structure of this industry and on methods to provide LTC effectively and efficiently. One potentially productive approach is to examine multiple national systems of LTC in order to understand better the effects of particular designs., ${ }^{4,5}$ In this vein, statistics have been examined that compare LTC costs, beds per 1000 elderly, nursing home occupancies, and the like. Intrinsic in such comparisons is an assumption that the basic structures are consistent across international boundaries, that is that a nursing home operates the same in one country as in another. Thus, while acknowledging on the one hand that LTC systems are different-the motivation to compare them in the first place-we nevertheless discuss them using terms such as "nursing home" that are implicitly assumed to be invariant.

While acute care hospitals are relatively well defined,

From the *Institute of Gerontology and School of Public Health, The University of Michigan, and Geriatric Research, Education, and Clinical Center, Ann Arbor Veterans' Administration Medical Center, Ann Arbor, Michigan; the †Department of Geriatric Medicine, Karolinska Institute, and Center for Health Economics, Stockholm, Sweden; and the †Department of Geriatric Medicine, Karolinska Institute, and Stockholm Gerontology Research Center, Stockholm, Sweden

Preliminary versions of this paper was presented at the Fourteenth (XIV) World Conference of Gerontology in Acapulco, Mexico in June, 1989 , and at the Gerontological Society of America meeting in Minneapolis, Minnesota in November 1989.

Address correspondence to Brant $\mathrm{E}$. Fries, PhD, Institute of Gerontology, The University of Michigan, 300 North Ingalls, Ann Arbor, Michigan 48109-2007. we see a variety of names designating LTC facilities. For example, in the United States (US) the following terms at least in part are overlapping: nursing homes; skilled nursing, intermediate care, extended care, residential care, and adult foster care facilities; and rehabilitation hospitals.

We suggest here a potential solution to this problem. While the structure of a health care system explicitly and implicitly defines its components (such as nursing facilities), the characteristics of the residents of these facilities are less affected. If we can define classifications of residents or patients in a health care system that are independent of the source of their care, then these classifications are valid across national boundaries. Further, defining a facility in terms of its residents avoids the problems of nomenclature and can allow valid cross-national comparisons.

The purpose of this study is two-fold. We demonstrate the feasibility of applying a resident-level classification system to comparing nursing homes in two countries, and we use this methodology to demonstrate significant differences between a sample of Swedish LTC institutions and those in the United States.

\section{NURSING HOME RESIDENT CLASSIFICATION}

The key to the approach suggested here is the availability of a classification system for long-term care that can define, reliably and validly, differences between individual residents. Given the focus on effectiveness and efficiency, it is important that such a system also be descriptive of resources. The resources to be considered should include not only money but all health care professionals, supplies, buildings, etc.; all are scarce resources. 
During the past decade considerable attention has been given to classifying patients in acute care hospitals and to understanding the level of resources used in their care. Such case-mix systems have been useful in determining payment, monitoring quality of care, and facility management. The best known hospital patient classification system is the Diagnosis-Related Groups ${ }^{6}$ (DRGs), although many other systems have been suggested. $^{7-9}$ The DRGs are the basis in the US for paying hospitals under Medicare and are increasingly being used for international comparisons. ${ }^{10}$ For LTC, however, there are considerably fewer resident classification systems, especially those that link resident characteristics directly with resource use. ${ }^{11-15}$ Resident-centered case mix is incorporated into the nursing home payment systems of several US states (eg, Minnesota, ${ }^{16}$ Maryland ${ }^{17}$ Washington ${ }^{18}$ ); several of these have been compared by Fries. ${ }^{19}$

We focus here on one that has undergone significant derivation and validation in the US: Resource Utilization Groups (RUG-II). ${ }^{20}$ The RUG-II system conceptually has its roots in the DRGs but also manifests significant differences necessary for LTC. Like DRGs, RUG-II represents a classification system, with residents in a group having similar resource consumption. The derivation of both systems was accomplished with an interactive form of cluster analysis ${ }^{21,22}$ to produce a set of mutually exclusive and exhaustive groups that are clinically sensible and best explain resource use. However, the similarity ends there. First, with highly variable lengths of stay, RUGs cannot feasibly consider the full cost of episodes (as with DRGs) but rather explain per diem costs. ${ }^{20,23}$ Those costs found to vary significantly across residents were primarily for nursing staff together with occupational, physical, and speech therapists and social workers. Other costs were relatively smaller (eg, special diets), unlikely to vary across residents (eg, building maintenance or capital costs), or likely to be correlated with other costs already explained by case mix (eg, pharmacy). Second, the resource needs of the elderly are associated more closely with functional capacity than diagnoses. As a result, the RUG-II system relies heavily, but not exclusively, on the Activities of Daily Living (ADLs). ${ }^{24}$

The RUG-II system consists of 16 groups of residents determined by two major constructions..$^{20}$ Residents are first categorized into five mutually exclusive groups representing their major type: Heavy Rehabilitation, Special Care, Clinically Complex, Severe Behavioral, and Reduced Physical Functions (Table 1). In addition, an ADL Index value is computed based upon the resident's assessed functioning in toileting, eating, and bed/chair transfer (Table 2). This index takes on values from 3 (totally independent) to 10 (totally dependent

\section{TABLE 1. CRITERIA FOR CLASSIFYING RESIDENTS INTO MAJOR RUG-II CATEGORIES}

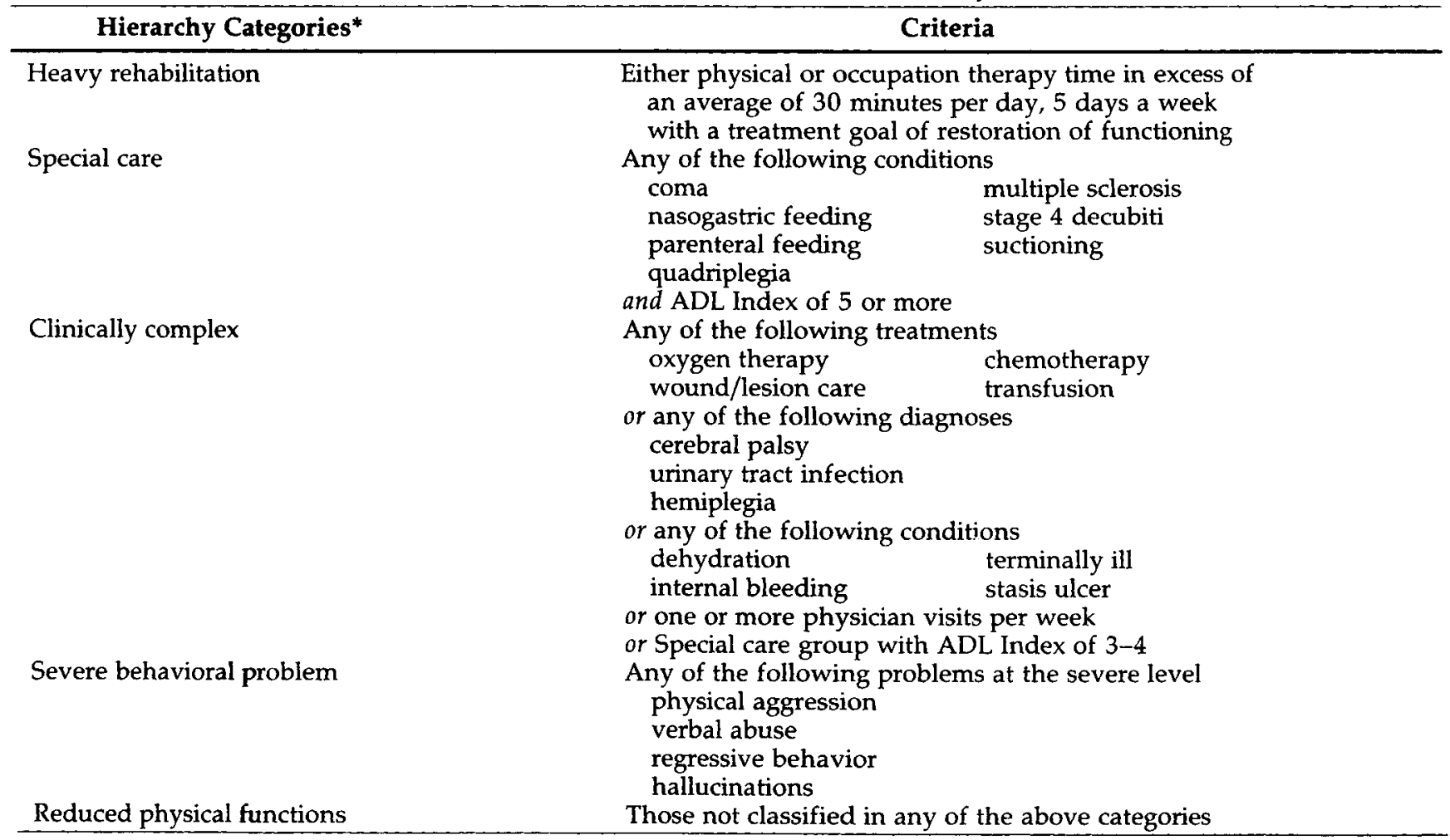

* Residents are classified into the first of the five listed categories into which they fit. Residents not classified into any of the first four categories are automatically assigned into the last, Reduced Physical Function. 
nursing home payment in New York and Texas and will soon be implemented by at least six more states. We are currently evaluating its application to a sample of residents in Swedish nursing facilities to demonstrate its validity and possibly to determine Swedish CMIs. ${ }^{25}$ To our knowledge, the current project represents the first use of such a system for international comparisons.

\section{METHODOLOGY}

Samples A survey of 1,134 residents in LTC facilities of Stockholm County was performed in 1987 to classify each resident into a RUG-II group. The residents were the total population of six LTC institutions assessed once: one department of geriatrics (146 patients); three nursing homes (two with 100 residents and one with 150 residents); and two mixed institutions (250 and 380 residents) consisting of a combination of geriatric wards and nursing home wards. In total, the sample represents $12.5 \%$ of all Stockholm LTC beds in 1987. In Sweden, each LTC organization (sometimes divided into nursing homes and geriatrics departments) has a defined area-based elderly population for which it solely is responsible, and the residents it cares for represent all LTC institutional use of this population.

The Swedish data were contrasted with those from New York State (NYS). This second "sample" is the entire population of NYS nursing home residents, assessed for payment determination and quality assurance. Included are both levels of US nursing facilities: intermediate care facilities (ICF), denoted health-related facilities in New York State, and skilled nursing facilities (SNFs). New York State has approximately 100,000 nursing home residents, two-thirds of which are in SNFs. All residents in a facility are assessed twice a year, with new admissions included at the end of their first quarter in the nursing home. The data set used here represents a cross section of the population collected in a single wave of assessments from July to December, 1988. In total, there were 94,840 residents assessed, with $73,118(77 \%)$ of these in SNF units. Although the level of resources in New York nursing facilities is higher than in most other states, previous research has shown that relative differences between resident groups are constant.

Determination of RUG-II Category The RUG-II category can be derived by assessing approximately 15 items. Alternatively, the resident's RUG-II group can be determined directly by a trained professional, applying the rules described in the previous section. In this study, the Swedish assessments were performed in the latter manner. A short manual of the RUG-II system was distributed, and a form was filled in by each head nurse during a specific week indicating the RUG-II category for each resident currently on the unit. In New York, RUG-II categories were obtained from the ongoing resident assessment process and the application of a computerized algorithm, both integral to the case-mix payment system.

Analysis The distribution of residents in the two samples was contrasted. As will be seen, the results depend strongly upon which of the NYS populations is considered, ICF or SNF. Comparisons were made at the level of the five major RUG-II categories, across the ADL Index, as well as by RUG-II category. Chisquared tests of significance were applied to the distribution to test their homogeneity, and two-sample parametric tests were applied for other comparisons. All results discussed in the following were significant at least at the $p=.01$ level unless otherwise noted. With the large sample sizes involved in this study, almost any comparison will be significant, so the analysis focuses more on the magnitude of differences than on their statistical significance.

\section{RESULTS}

We began by comparing the ADL Index values (see Figure 1). Significant differences were seen between the New York State and Stockholm data. Only $18 \%$ of the Swedish nursing home residents were functionally independent in the three ADLs (with an Index value of 3). In contrast, more than a quarter of all NYS residents (ICF and SNF) were similarly independent, but this is primarily the result of over $80 \%$ of the ICF residents being assessed at that level. At the other end of the spectrum, fewer totally dependent residents (those with ADL Index values of at least 9) were seen in Sweden: 4\% compared with 7\% for all NYS facilities (and virtually all of the latter in SNFs). The highest index value represents residents totally dependent in three ADLs and tube-fed. While a very small percentage of these residents was seen in NYS $(0.2 \%)$, none were seen in Stockholm (difference not significant at $p$ $=.10$ ). This agrees with previous results obtained in transcultural comparisons by Norberg and colleagues, ${ }^{26,27}$ showing that Swedish nursing facilities do not usually care for tube-fed patients due to ethical considerations and concerns for quality of care.

Overall, the distribution of ADL Index in Stockholm was different from any of the patterns in New York (SNFs, ICFs, or all residents). Compared with the patterns of New York SNF residents, it was shifted toward lower dependencies, but compared with all New York residents (not shown), it was shifted toward more intermediate levels of functioning. As a result, the Stockholm average ADL Index (5.79) was substantially lower than that for New York SNFs (6.35) but close to the overall New York average (5.66).

Examination of the major categories of residents also indicated differences among the populations (see Table 4). The Stockholm nursing facilities care for over twice the proportion of residents that were classified as 


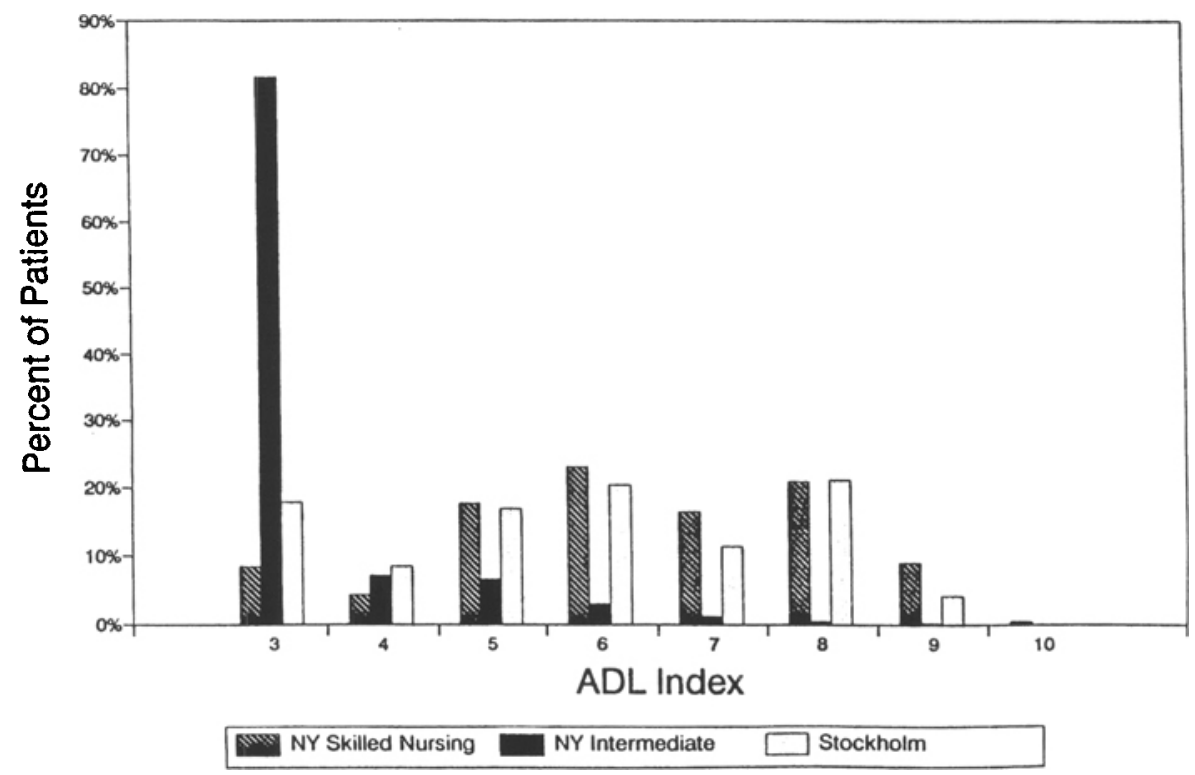

Figure 1. Distribution of ADL Index values for Stockholm and two levels of New York nursing facilities.

Heavy Rehabilitation compared to all those in NYS and over three-quarters more than NYS SNFs. Even bigger differences are seen for residents classified as having severe behavior problems, with over five times the proportion of residents in this category in Stockholm. In both NYS and Stockholm the Reduced Physical Functions category was the largest, but in NYS it represented $66 \%$ of all residents $(84 \%$ of the ICF residents), while in the Stockholm sample it represented only $45 \%$. However, combining the Severe Behavioral and Reduced Physical Functions groups, both samples had similar percentages of residents $(72 \%$ for all $\mathrm{New}$ York's residents versus $77 \%$ for Stockholm's). Thus, both populations have close to the same percentage of residents with severe or complex medical problems or receiving rehabilitation, while of those not receiving these services, a much larger portion in Stockholm are institutionalized for severe behavior problems, presumably due to dementia.

Together, all of these effects caused significant variations in the proportions in each RUG-II group (see Table 4). First, the distribution of New York ICF residents was markedly different from that for Stockholm, with $70 \%$ of the former in the lightest care (PA) category. For many groups, the proportions of Stockholm residents and those in NYS SNFs were similar, although for others substantial differences were observed. All three Severe Behavioral groups and the Heavy Rehabilitation-A group (RA) were more prevalent in the Stockholm sample, while all but one each of the Reduced Physical Functions and the Clinically Complex groups were more frequent in the NYS SNF data.

Overall, the average case mix index for all the residents in Stockholm was 1.10, significantly greater than the overall average of 1.04 for NYS but also lower than the New York SNF average of 1.16. The CMI for the six institutions in Stockholm ranged from 1.03 to 1.16 and for different wards from 0.93 to 1.52 . For comparative purposes, in New York State the CMI for the intermediate care facilities was 0.66 . No intermediate care facility in New York State had a CMI of more than 0.90 .

\section{DISCUSSION}

Three major findings emerge from our results, demonstrating important differences between the populations studied. First, Stockholm LTC residents not requiring either rehabilitation or major medical care are much more likely to have severe behavior problems. Although it is possible that in the data collection different interpretations were made of the severity of behavioral disturbances, the consistent methodologies and instructions used, the reliability testing, and the large differences seen make this unlikely. We expect that this result stems from two causes. Compared to the US, Sweden has a better developed home-based LTC system and may be more effective in hospital discharge planning. Also, within the last five to six years, Sweden has moved its hospitalized dementia patients to nursing home settings.

Second, the higher prevalence in Stockholm of rehabilitation patients, especially those with better ADL functionality, may in part be due to the still relatively small number of Swedes in programs where they are intermittently institutionalized for intensive rehabilitation on a bi-weekly or monthly schedule. In the US, many of these residents are funded by the time-limited benefits of Medicare and are discharged rapidly. Another explanation is a shortage of orthopedic beds in 
TABLE 4. RUG-II DISTRIBUTION OF NURSING HOME RESIDENTS AND AVERAGE CASE MIX IN NEW YORK STATE AND STOCKHOLM*

\begin{tabular}{|c|c|c|c|c|}
\hline \multirow{2}{*}{ RUG-II Groups } & \multicolumn{3}{|c|}{ New York State } & \multirow{2}{*}{ Stockholm } \\
\hline & Skilled Nursing & Intermediate Care & All & \\
\hline & $\%$ & $\%$ & $\%$ & $\%$ \\
\hline Heavy Rehabilitation & 5.1 & 1.6 & 4.3 & 9.1 \\
\hline $\mathrm{RA}^{\circ}$ & 0.6 & 1.2 & 0.7 & 5.7 \\
\hline $\mathrm{RB}$ & 4.6 & 0.4 & 3.6 & 3.4 \\
\hline Special Care & 6.8 & 0.1 & 5.3 & 4.2 \\
\hline SA & 1.5 & 0.1 & 1.2 & 1.0 \\
\hline SB & 5.3 & 0.0 & 4.1 & 3.3 \\
\hline Clinically Complex & 21.2 & 10.3 & 18.7 & 10.0 \\
\hline $\mathrm{CA}$ & 1.1 & 8.0 & 2.7 & 2.3 \\
\hline $\mathrm{CB}$ & 10.1 & 2.1 & 8.3 & 3.8 \\
\hline $\mathrm{CC}$ & 8.1 & 0.2 & 6.3 & 3.6 \\
\hline $\mathrm{CD}$ & 1.8 & 0.0 & 1.4 & 0.4 \\
\hline Severe Behavioral & 5.8 & 3.8 & 5.4 & 32.0 \\
\hline BA & 0.6 & 2.7 & 1.1 & 4.8 \\
\hline $\mathrm{BB}$ & 3.7 & 1.1 & 3.1 & 19.6 \\
\hline $\mathrm{BC}$ & 1.5 & 0.0 & 1.2 & 7.5 \\
\hline Reduced Physical Functions & 61.0 & 84.1 & 66.3 & $\mathbf{4 4 . 7}$ \\
\hline PA & 6.4 & 70.0 & 21.0 & 6.6 \\
\hline PB & 2.7 & 5.8 & 3.4 & 3.6 \\
\hline PC & 35.6 & 8.0 & 29.3 & 23.0 \\
\hline PD & 12.6 & 0.3 & 9.8 & 10.4 \\
\hline $\mathrm{PE}$ & 3.7 & 0.1 & 2.9 & 1.1 \\
\hline Totals & 99.9 & 100.0 & 100.1 & 100.1 \\
\hline Average case mix index & 1.16 & 0.66 & 1.04 & 1.10 \\
\hline
\end{tabular}

* Totals are not exact due to rounding.

Sweden, resulting in the lightest care rehabilitation residents being admitted to LTC institutions for care.

The last finding is that the Stockholm LTC facilities serve a more disabled population than that of the average American LTC facility, at least as represented by New York State's population. Stockholm facilities are akin to SNFs in New York both in average case mix and, with some notable exceptions, in the distribution of residents. We need to be cautious in generalizing this last finding to all US SNFs as this designation is used differently across the fifty states. For example, all facilities in Arizona are identified as SNFs, while only $2 \%$ of those in Oklahoma are similarly designated, ${ }^{28}$ yet there is no reason to believe that the overall nursing home populations in these two states differ. The ADL measures support the proposition that the least careintensive residents seen in US facilities are not in Swedish nursing homes, likely a result of the betterdeveloped home care system mentioned earlier and more active hospital discharge planning.

In any cross-national study such as this, there is always the possibility of intrinsic bias caused by the methodology employed, such as differences in the two data collections or the language or method by which data are collected. Although it is impossible to eliminate this possibility, several aspects of the data used suggest that such bias, if it occurred, does not explain the described findings. The New York data, in addition to being extensive in scope, was collected to determine case-mix-adjusted payment to nursing homes. As part of this process, there is a stringent audit process that focuses on specific residents and particular conditions that are associated with higher CMI and increased payments. Thus, although there is an implicit incentive to over-report conditions or ADL functional limitations that would categorize residents into higher RUG-II groups, this practice is carefully controlled. In any case, if such over-reporting had occurred, it would have had the overall effect of diminishing our observed differences rather than causing them. Also, the data presented are comprehensive to the entire nursing home population for the selected areas: all of New York State and all nursing homes providing care for a significant portion of Stockholm's population. Thus, we avoid the difficulties of comparisons that would contrast certain types of nursing homes, such as geriatric units in Stockholm. Finally, case-mix has been changing slowly over time in New York, in part as a result of the implementation of a case-mix payment system that provides increased revenue for heavier care residents. Thus, the use of 1988 data in NYS to contrast with 1987 data from Stockholm provides a conservative comparison of case mix.

There is also reason to be optimistic that the RUG-II system captures well characteristics of nursing facility residents in both countries. Preliminary findings in a new validation study support the appropriateness of the RUG-II classification system as a measure of re- 
source use in Sweden. ${ }^{25}$ There are also more structural reasons to trust this application, relying upon the clinical rationale of the RUG-II system and its strong involvement of the ADLs. ADLs have been shown to be predictive of resource use in a range of settings. In addition, the RUG-II system has been shown to be robust, at least in the US, across a range of long-term care settings including home health care and post-acute care. ${ }^{23,29}$ However, there may be transcultural differences in the content of care between the two countries, differences that may influence resource allocation and staffing, and thereby affect the appropriate CMI to be used.

\section{CONCLUSION}

The application of a consistent methodology of classifying nursing home residents demonstrated significant differences in the nursing home populations in a large US state and Stockholm. Such an approach provides a method to contrast health care organizations, at least partly independent of how they are named in different languages and how they operate in different countries. Overall, the Stockholm nursing facilities care for a relatively heavier case load (as measured by relative resource use) than the average New York facility and are more like skilled nursing facilities or extended care units. Whether these differences are due to practice patterns, financing levels or payment methodologies, epidemiological differences in the two populations, or a variety of other causes is beyond the scope of the current study. These are obvious opportunities for further investigations.

International comparisons provide the possibility of examining how the organization of long-term care affects the well-being of the elderly. As the US and other nations move toward a mandated uniform assessment of nursing home residents, ${ }^{30}$ the type of comparative work described here will become increasingly more feasible and accurate. We suggest that the methodology employed is feasible for cross-national studies of long-term care in order to learn from the broad spectrum of approaches employed across the world to care for our growing elderly population.

\section{ACKNOWLEDGMENTS}

We acknowledge gratefully the assistance of Marie Gavazzi of the New York State Department of Health and Don Schneider of Rensselaer Polytechnic Institute in obtaining the case-mix data for New York State, the helpful comments of three anonymous reviewers, and the editorial care of the Editor.

\section{REFERENCES}

1. Stout RW, Crawford V: Active-life expectancy and terminal dependency: trends in long-term geriatric care over 33 years. Lancet 1:281, 1988

2. Winblad B, Ljunggren G: Life expectancy and planning care for the elderly. Letter. Lancet 11:1313, 1988
3. Doty P, Liu K, Wiener J: An overview of long-term care. Health Care Financing Review 6:69, 1985

4. Doty P: Long-term care in international perspective. Health Care Financing Review, Annual supplement 145, 1988

5. Berg S, Branch LG, Doyle AE, et al: Institutional and homebased long-term care alternatives: The 1965-1985 Swedish experience. Gerontologist 28:825, 1988

6. Fetter RB, Shin Y, Freeman JL, et al: Case mix definition by diagnosis-related groups. Med Care (Phila) 18 (Supplement), 1980

7. Horn SD, Horn RA: Reliability and validity of the severity of illness index. Med Care (Phila) 24: 159, 1986

8. Brewster AC, Karlin BG, Hyde LA, et al: MEDISGRPS: A clinically based approach to classifying hospital patients at admission. Inquiry 22:377, 1985

9. Jencks SF, Dobson A: Refining case-mix adjustment: the research evidence. N Engl J Med 317:679, 1987

10. Håkansson $S$, Paulson $E$, Kogeus $K$ : Prospects for using DRGs in Swedish hospitals. Health Policy 9:177, 1988

11. Arling G, Nordquist RH, Brant BA, et al: Nursing home case mix: patient classification by nursing resource use. Med Care (Phila) 25:9, 1987

12. Arling G, Zimmerman D, Updike L: Nursing home case mix in Wisconsin. Med Care (Phila) 27:164, 1989

13. Cameron JM: Case mix and resource use in long-term care. Med Care (Phila) 23:296, 1985

14. Fries BE, Cooney LM: Resource utilization groups: a patient classification system for long-term care. Med Care (Phila) 23:110-122, 1985

15. Morris JN, Sherwood S, May MI, et al: FRED(c): An innovative approach to nursing home level-of-care assignments. Health Serv Res 22(1):118-138, 1987

16. Minnesota Department of Health: Facility manual for completing case mix requests for classification. Minneapolis, MN, 1986

17. Deane RT, Cella MA: New concepts in nursing home reimbursement. Silver Springs, MD, Applied Management Sciences, 1981

18. McCaffree K, Winn S, Bennett CA: Final report of cost data reporting system for nursing home care. Seattle, WA, Battelle Human Affairs Research Center, 1976

19. Fries BE: Comparing case-mix systems for nursing home payment. Health Care Financing Review 11:103, 1990

20. Schneider DP, Fries BE, Foley W], et al: Case mix for nursing home payment: Resource utilization groups, version II. Health Care Financing Review, Annual Supplement 39, 1988

21. Morgan JN, Sonquist JA: Problems in the analysis of survey data and a proposal. J Am Stat Assoc 58:415, 1963

22. Mills R, Fetter RB, Riedel DC, et al: AUTOGRP: An interactive computer system for the analysis of health care data. Med Care (Phila) 14:603, 1976

23. Fries BE, Schneider DP, Foley WJ, et al: Case-mix classification of medicare residents in skilled nursing facilities: Resource Utilization Groups (RUG-T18). Med Care (Phila) 27:843, 1989

24. Katz $\mathrm{S}$, Form $A B$, Moskowitz RW, et al: Studies of illness in the aged: the index of ADL: a standardized measure of biological and psychosocial function. JAMA 195:914-919, 1963

25. Ljunggren $G$, Fries $B E$, Winblad $B$ : International validation of a resource measurement technology in long-term care. Presented at the Sixth Annual Meeting of the International Society for Technology Assessment in Health Care, Houston, TX, May 1990

26. Norberg A, Hirschfeld M: Feeding of severely demented patients in institutions: interviews with caregivers in Israel. J Adv Nurs $12: 551,1987$

27. Norberg A, Bäckström A, Athlin E, et al: Food refusal amongst nursing home patients as conceptualized by nurses' aides and enrolled nurses: an interview study. J Adv Nurs 13:478, 1988

28. Institute of Medicine: Improving the quality of care in nursing homes. Washington, DC, National Academy Press, 1986

29. Foley WJ, Schneider D, Dowling $M$, et al: Development of a survey, case mix measurement system, and assessment instrument to rationalize the long term care home care system. Final report on contract \#12,000 C001558 to New York State Department of Health. Troy, NY, Rensselaer Polytechnic Institute, 1986

30. Morris JN, Hawes C, Fries BE, et al: Designing the national resident assessment instrument for nursing facilities. Gerontologist 30:293, 1990 